\title{
A stabilized coupled method and its optimal error estimates for elliptic interface problems
}

\author{
Jiaping $\mathrm{Yu}^{1,2}$, Feng Shi ${ }^{3^{*}}$ and Jianping Zhao ${ }^{4,5}$
}

"Correspondence: shi.feng@hit.edu.cn

${ }^{3}$ College of Science, Harbin Institute of Technology, Shenzhen, China Full list of author information is available at the end of the article

\begin{abstract}
In this paper, we present a stabilized coupled algorithm for solving elliptic interface problems, mainly by introducing the jump of the solutions along the interface. A framework of theoretical proofs is provided to show the optimal error estimates of this stabilized method. Several numerical experiments are carried out to demonstrate the computational stability and effectiveness of the method.
\end{abstract}

Keywords: The interface problem; Stabilized method; Couple method; Optimal error estimates

\section{Introduction}

The interface problem has always been a difficult issue in multi-physics and multi-phase applications in science and engineering and has becoming focused recently. In real physical world, many phenomena need to be described using non-smooth or even discontinuous functions/methods. A lot of methods have been put forward to improve the standard numerical methods which may not be applied directly. Focusing on the approximation of non-smooth solutions related to the present work, there are mainly two fundamentally different approaches.

One approach of improvement is to enrich the approximation space or discrete form, for instance, the immersed boundary method (IBM) [1], the immersed interface method (IIM) [2-5] and some modified methods for them in the finite difference method (FDM). This type of methods usually incorporates the interface conditions into the finite difference scheme near the interface to achieve second- or higher-order accuracy based on a Taylor expansion in a local coordinate system. Concentrated on the development of the highaccuracy or augmented method [6], these methods can treat the irregular domain problem on a rectangular domain so that fast solvers for Poisson/Helmholtz equations can be used. Several methods of finite element versions, such as the immersed interface finite element method (IIFEM) [7, 8], the weak Galerkin finite element method [9], the extended finite element method (XFEM) [10,11], the generalized finite element method (GFEM) [12], and non-traditional finite element methods $[13,14]$ have also been developed. These methods usually modify the basis function or add virtual nodes near the interface.

Another approach of improvement is to refine the discretization near the critical regions, so the procedure of re-meshing is usually required in this case. For instance, by placing more grid-points along the interface and around the intersection. Bernardi and

(c) The Author(s) 2019. This article is distributed under the terms of the Creative Commons Attribution 4.0 International License (http://creativecommons.org/licenses/by/4.0/), which permits unrestricted use, distribution, and reproduction in any medium, provided you give appropriate credit to the original author(s) and the source, provide a link to the Creative Commons license, and indicate if changes were made. 
Figure 1 Schematic diagram of problem domain

$$
\Omega_{1}
$$

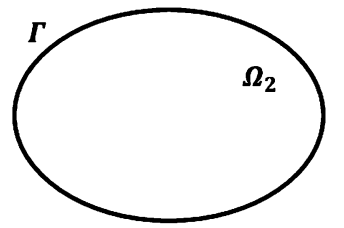

Verfurth proposed weighted-residual error estimators to deal with interfaces [15] and Cai and Zhang proposed recovery-based error estimators [16, 17]. Note that, in the previous method, the meshes were generated along the interface.

For many numerical methods, researchers often use stabilization to reduce the error [18], which inspires us to solve the interface problem in such way. By combining the two-level method and the partition of unity, the authors and their collaborators proposed two local and parallel algorithms for the Stokes problem [19], elliptic equations [20], the Stokes-Darcy model [21] and the fluid-fluid model [22, 23]. In this paper, we consider the elliptic interface problem as a mixed elliptic-elliptic model. We will introduce the jump of the solution along the interface as our stabilization term of the stabilized method and then obtain the optimal error estimates for the method.

The rest of the article is organized as follows. In Sect. 2, some preliminary notations and inequalities are introduced. As the main parts of this paper, in Sect. 3 and 4, the stabilized coupled algorithm and its analysis are discussed. Then numerical tests are presented in Sect. 5. Finally, some conclusions are given in Sect. 6.

\section{Preliminaries}

In this section, we will introduce some basic notations and inequalities. Let $\Omega \subset \mathbf{R}^{d}$ ( $d=$ $2,3)$ be a bounded domain with Lipschitz boundary and $\Omega=\Omega_{1} \cup \Gamma \cup \Omega_{2}$. Here $\Omega_{1}$ and $\Omega_{2}$ are two disjoint subdomains which are either convex or of class $C^{1,1}$, and coupled by a fixed interface $\Gamma \subseteq \partial \Omega$ (see Fig. 1 for example). Both boundaries $\partial \Omega_{1}, \partial \Omega_{2}$ and interface $\Gamma$ are assumed to be polygonal $(d=2)$ or polyhedral $(d=3)$ for the sake of avoiding analyzing the effect caused by triangulation of domain into finite elements [24]. For any domain $D$, we denote the norm for the Sobolev space $H^{k}(D)$ by $\|\cdot\|_{k, D}$ with $k>0$ (see for instance [25]). When $k=0$, we denote $W^{0, m}(D)$ connected with the norm $\|\cdot\|_{L^{m}(D)}$ by $L^{m}(D)$. In particular, $W^{0,2}(D)$ with the norm $\|\cdot\|_{0, D}$ is denoted by $L^{2}(D)$. The definitions of the space $H_{00}^{\frac{1}{2}}(\Gamma)$ and its dual space $H_{00}^{-\frac{1}{2}}(\Gamma)$ will also be used and are referred to [26].

We then define two spaces by

$$
X_{i}=\left\{v_{i} \in H^{1}\left(\Omega_{i}\right) ;\left.v_{i}\right|_{\partial \Omega_{i} \backslash \Gamma}=0\right\}, \quad i=1,2,
$$

and their tensor space by

$$
X:=X_{1} \times X_{2}
$$


For $i=1,2$, let $\pi_{h}^{i}$ be a regular partition of each subdomain $\Omega_{i}$, which consists of $K$ that are tetrahedral elements for $d=3$ and triangles for $d=2$. We denote the diameter of the element $K \in \pi_{h}=\pi_{h}^{1} \cup \pi_{h}^{2}$ by $h_{K}$ and mesh scale $h=\max _{K \in \pi_{h}} h_{K}$. Accordingly, we define the following finite element spaces on $\pi_{h}^{i}$ :

$$
X_{i, h}=X_{i} \cap\left\{v_{i, h} \in H^{1}\left(\Omega_{i}\right) ;\left.v_{i, h}\right|_{K} \in P_{k}(K), K \in \pi_{h}^{i}\right\} \subset X_{i}, \quad i=1,2,
$$

where $P_{k}(K)$ is the space of polynomials of order $k$ on $K$ with $k \geq 1$, and tensor space

$$
X_{h}:=X_{1, h} \times X_{2, h} \subset X
$$

Some useful inequalities are listed in the end of this section. We refer to $[26,27]$ for such inequalities.

A1: (Trace inequality) For any $v_{i} \in X_{i}$,

$$
\left\|v_{i}\right\|_{\Gamma} \leq \tilde{C}_{\mathrm{tr}}\left\|v_{i}\right\|_{0}^{\frac{1}{2}}\left\|v_{i}\right\|_{1}^{\frac{1}{2}}
$$

A2: (Interpolation inequalities) For any $u_{i} \in X_{i} \cap H^{k+1}\left(\Omega_{i}\right)$ with $k \geq 1$, there exists $I_{h} u_{i} \in X_{i, h}$ such that

$$
\left\|u_{i}-I_{h} u_{i}\right\|_{0, \Omega_{i}}+h\left\|u_{i}-I_{h} u_{i}\right\|_{1, \Omega_{i}} \leq C_{I} h^{k+1}\left\|u_{i}\right\|_{k+1, \Omega_{i}}
$$

\section{The elliptic interface problem}

The model of the elliptic interface problem considered in this paper is as follows:

$$
\begin{aligned}
& -\nabla \cdot\left(v_{i} \nabla u_{i}\right)=f_{i}, \quad \text { in } \Omega_{i}, i=1,2, \\
& u_{i}=0, \quad \text { on } \partial \Omega_{i} \backslash \Gamma, \\
& u_{1}-u_{2}=0, \quad \text { on } \Gamma, \\
& n_{1} \cdot v_{1} \nabla u_{1}+n_{2} \cdot v_{2} \nabla u_{2}=g, \quad \text { on } \Gamma .
\end{aligned}
$$

Here for $i=1,2, n_{i}$ is the unit outward normal to $\Gamma$ of domain $\Omega_{i}, f$ is the given source term, and $u_{i}$ is the restriction of $u$ in $\Omega_{i}$.

The variational formulation of the problem (3)-(6) is: for any given $h$, find $u_{i} \in X_{i}$ satisfying

$$
\begin{aligned}
a_{i}\left(u_{i}, v_{i}\right) & +\frac{\delta}{h} \int_{\Gamma}\left(u_{1}-u_{2}\right)\left(v_{1}-v_{2}\right) d s \\
& -\frac{1}{2} \int_{\Gamma}\left(v_{1} \nabla u_{1} \cdot n_{1}-v_{2} \nabla u_{2} \cdot n_{2}\right)\left(v_{1}-v_{2}\right) d s \\
= & \left(f_{i}, v_{i}\right)+\frac{1}{2} \int_{\Gamma} g v_{1} d s+\frac{1}{2} \int_{\Gamma} g v_{2} d s, \quad \forall v_{i} \in X_{i}, i=1,2,
\end{aligned}
$$

where

$$
a_{i}\left(u_{i}, v_{i}\right)=\left(v_{i} \nabla u_{i}, \nabla v_{i}\right)_{\Omega_{i}} .
$$


Since in the present situation $u_{1}-u_{2}=0$ on $\Gamma$, we actually add a zero term $\frac{\delta}{h} \int_{\Gamma}\left(u_{1}-\right.$ $\left.u_{2}\right)\left(v_{1}-v_{2}\right) d s$ in the variational formulation to (3)-(6) comparing with the standard weak formulation. However, this modification will be very useful and important as shown in our later analysis in the next section and also numerical simulations.

By introducing some important notations for $\mathbf{u}=\left(u_{1}, u_{2}\right), \mathbf{v}=\left(v_{1}, v_{2}\right) \in X$

$$
\begin{aligned}
& {[\mathbf{u}]=\left.\left(u_{1}-u_{2}\right)\right|_{\Gamma},} \\
& a(\mathbf{u}, \mathbf{v})=a_{1}\left(u_{1}, v_{1}\right)+a_{2}\left(u_{2}, v_{2}\right), \\
& \left(u_{i}, v_{i}\right)_{\Gamma}=\int_{\Gamma} u_{i} \cdot v_{i} d s, \\
& (g, \mathbf{v})=\left(g, v_{1}\right)_{\Gamma}+\left(g, v_{2}\right)_{\Gamma},
\end{aligned}
$$

the above variational formulation (7) can be equivalently rewritten as: Find $\mathbf{u} \in X$ satisfying

$$
a(\mathbf{u}, \mathbf{v})+\frac{\delta}{h}([\mathbf{u}],[\mathbf{v}])_{\Gamma}+\frac{1}{2}([v \nabla \mathbf{u} \cdot \mathbf{n}],[\mathbf{v}])_{\Gamma}=(\mathbf{f}, \mathbf{v})+\frac{1}{2}(g, \mathbf{v}), \quad \forall \mathbf{v} \in X .
$$

We also need the inverse and trace inequality as follows [26, 27]:

$$
\left\|v_{i, h}\right\|_{\Gamma} \leq \tilde{C}_{\mathrm{inv}} h^{-1 / 2}\left\|v_{i, h}\right\|_{0}, \quad \forall v_{i, h} \in X_{i, h}
$$

\section{The coupled algorithm for the interface model and error estimates}

In this section, we will present our algorithm for solving the coupled problem (3), which is referred as the coupled scheme.

Algorithm 1 (The coupled scheme) Find $u_{i, h} \in X_{i, h}, i=1,2$, such that

$$
\begin{aligned}
\sum_{i=1}^{2} a_{i}\left(u_{i, h}, v_{i, h}\right)+\frac{\delta}{h} \int_{\Gamma}\left(u_{1, h}-u_{2, h}\right)\left(v_{1, h}-v_{2, h}\right) d s & \\
& -\frac{1}{2} \int_{\Gamma}\left(v_{1} \nabla u_{1, h} \cdot n_{1}-v_{2} \nabla u_{2, h} \cdot n_{2}\right)\left(v_{1, h}-v_{2, h}\right) d s \\
= & \sum_{i=1}^{2}\left(f_{i}, v_{i, h}\right)+\frac{1}{2} \sum_{i=1}^{2} \int_{\Gamma} g v_{i, h} d s, \quad \forall v_{i, h} \in X_{i, h} .
\end{aligned}
$$

Remark 1 In the present work, to keep the briefness of introducing our coupled method and proving its optimal error estimates, we only consider both homogeneous noninterface boundary condition (4) and homogeneous interface jump condition (5). The extension to the general conditions $u_{i}=\phi_{i}$, on $\partial \Omega_{i} \backslash \Gamma, u_{1}-u_{2}=\psi$ on $\partial \Gamma$ as studied in [9] is trivial. Actually, we only need modify two places in the numerical scheme (10): (i) adding $\frac{\delta}{h} \int_{\Gamma} \psi\left(v_{1, h}-v_{2, h}\right) d s$ in the right side of (10); (ii) replacing solution spaces $X_{i, h}$ by $\tilde{X}_{i, h}=\tilde{X}_{i} \cap\left\{v_{i, h} \in H^{1}\left(\Omega_{i}\right) ;\left.v_{i, h}\right|_{K} \in P_{k}(K), K \in \pi_{h}^{i}\right\}$, with $\tilde{X}_{i}=H^{1}\left(\Omega_{i}\right)$, and searching $u_{i, h} \in \tilde{X}_{i, h}$ with $\left.u_{i, h}\right|_{\partial \Omega_{i} \backslash \Gamma}=J_{i, h} \phi_{i}$, here $J_{i, h}$ are standard interpolation operator associated with $\pi_{h}^{i}$. The analysis in the following two theorems is nearly same for this non-homogeneous case. 
We can also equivalently rewrite this scheme as: Find $\mathbf{u}_{h} \in X_{h}$ satisfying

$$
a\left(\mathbf{u}_{h}, \mathbf{v}_{h}\right)+\frac{\delta}{h}\left(\left[\mathbf{u}_{h}\right],\left[\mathbf{v}_{h}\right]\right)_{\Gamma}+\frac{1}{2}\left(\left[v \nabla \mathbf{u}_{h} \cdot \mathbf{n}\right],\left[\mathbf{v}_{h}\right]\right)_{\Gamma}=\left(\mathbf{f}, \mathbf{v}_{h}\right)+\frac{1}{2}\left(g, \mathbf{v}_{h}\right), \quad \forall \mathbf{v}_{h} \in X_{h} .
$$

In the remaining part of this section, we will focus on deriving the optimal error estimates for the coupled Algorithm 1. Actually, Theorem 1 and Theorem 2 will provide the error estimates of the numerical solutions in $H^{1}$ norm and $L^{2}$ norm, respectively.

Theorem 1 Let $\mathbf{u}$ be the solution of the variational formulation (8) and $\mathbf{u}_{h}$ be the finite element solution deduced by Algorithm 1 . Under the hypothesis that $\mathbf{u} \in X \cap H^{k+1}(\Omega)$, the following estimate holds:

$$
\left\|\mathbf{u}-\mathbf{u}_{h}\right\|_{1, \Omega} \leq C h^{k}
$$

Proof For convenience, we separate the errors into two parts,

$$
u_{i}-u_{i, h}=\left(u_{i}-I_{h} u_{i}\right)+\left(I_{h} u_{i}-u_{i, h}\right):=\phi_{i}+\theta_{i}^{h}, \quad i=1,2 .
$$

Then by denoting $\Phi=\left(\phi_{1}, \phi_{2}\right), \Theta^{h}=\left(\theta_{1}^{h}, \theta_{2}^{h}\right)$, we obviously have

$$
\mathbf{u}-\mathbf{u}_{h}=\Phi+\Theta^{h}
$$

Subtracting (11) from (8) gives the error equations as

$$
a\left(\mathbf{u}-\mathbf{u}_{h}, \mathbf{v}_{h}\right)+\frac{\delta}{h}\left(\left[\mathbf{u}-\mathbf{u}_{h}\right],\left[\mathbf{v}_{h}\right]\right)_{\Gamma}+\frac{1}{2}\left(\left[v \nabla\left(\mathbf{u}-\mathbf{u}_{h}\right) \cdot \mathbf{n}\right],\left[\mathbf{v}_{h}\right]\right)_{\Gamma}=0, \quad \forall \mathbf{v}_{h} \in X_{h} .
$$

Then taking $\mathbf{v}_{h}=\Theta^{h}$ in (13) it follows

$$
\begin{aligned}
& a\left(\Theta^{h}, \Theta^{h}\right)+\frac{\delta}{h}\left(\left[\Theta^{h}\right],\left[\Theta^{h}\right]\right)_{\Gamma}+\frac{1}{2}\left(\left[v \nabla \Theta^{h} \cdot \mathbf{n}\right],\left[\Theta^{h}\right]\right)_{\Gamma} \\
& \quad=-a\left(\Phi, \Theta^{h}\right)-\frac{\delta}{h}\left([\Phi],\left[\Theta^{h}\right]\right)_{\Gamma}-\frac{1}{2}\left([v \nabla(\Phi) \cdot \mathbf{n}],\left[\Theta^{h}\right]\right)_{\Gamma} .
\end{aligned}
$$

Using the inverse inequality (9), the Hölder inequality and denoting two positive constants

$$
v_{\max }=\sup _{\mathbf{x} \in \Omega_{1}, \mathbf{y} \in \Omega_{2}}\left\{v_{1}(\mathbf{x}), v_{2}(\mathbf{y})\right\}, \quad v_{\min }=\inf _{\mathbf{x} \in \Omega_{1}, \mathbf{y} \in \Omega_{2}}\left\{v_{1}(\mathbf{x}), v_{2}(\mathbf{y})\right\},
$$

we can derive the following estimates for (14):

$$
\begin{aligned}
& \frac{1}{2}\left(\left[v \nabla \Theta^{h} \cdot \mathbf{n}\right],\left[\Theta^{h}\right]\right)_{\Gamma} \leq\left\|\left[v \nabla \Theta^{h} \cdot \mathbf{n}\right]\right\|_{\Gamma}\left\|\left[\Theta^{h}\right]\right\|_{\Gamma} \\
& \leq \tilde{C}_{\text {inv }} h^{-1 / 2}\left\|v \nabla \Theta^{h}\right\|_{0}\left\|\left[\Theta^{h}\right]\right\|_{\Gamma} \\
& \leq \frac{1}{4}\left\|\sqrt{v} \nabla \Theta^{h}\right\|_{0}^{2}+\frac{\tilde{C}_{\text {inv }}^{2} v_{\max }}{h}\left\|\left[\Theta^{h}\right]\right\|_{\Gamma}^{2}, \\
& a\left(\Phi, \Theta^{h}\right) \leq\|v \nabla \Phi\|_{0}\left\|\nabla \Theta^{h}\right\|_{0} \leq \frac{1}{4}\left\|\sqrt{v} \nabla \Theta^{h}\right\|_{0}^{2}+v_{\max }\|\nabla \Phi\|_{0}^{2},
\end{aligned}
$$




$$
\begin{aligned}
& \frac{\delta}{h}\left([\Phi],\left[\Theta^{h}\right]\right)_{\Gamma} \leq \frac{\delta}{h}\|[\Phi]\|_{\Gamma}\left\|\left[\Theta^{h}\right]\right\|_{\Gamma} \leq \frac{\delta}{2 h}\left\|\left[\Theta^{h}\right]\right\|_{\Gamma}^{2}+\frac{\delta}{2 h}\|[\Phi]\|_{\Gamma}^{2}, \\
& \frac{1}{2}\left([v \nabla(\Phi) \cdot \mathbf{n}],\left[\Theta^{h}\right]\right)_{\Gamma} \leq\|[v \nabla \Phi \cdot \mathbf{n}]\|_{\Gamma}\left\|\left[\Theta^{h}\right]\right\|_{\Gamma} \\
& \leq \tilde{C}_{\mathrm{inv}} h^{-1 / 2}\|v \nabla \Phi\|_{0}\left\|\left[\Theta^{h}\right]\right\|_{\Gamma} \\
& \leq \frac{v_{\max }}{4}\|\nabla \Phi\|_{0}^{2}+\frac{\tilde{C}_{\mathrm{inv}}^{2} v_{\max }}{h}\left\|\left[\Theta^{h}\right]\right\|_{\Gamma}^{2}
\end{aligned}
$$

Then combining (14) with (15)-(18), and applying the trace inequality A1, we can obtain

$$
\begin{aligned}
\frac{1}{2}\left\|\sqrt{v} \nabla \Theta^{h}\right\|_{0}^{2}+\frac{\delta-4 \tilde{C}_{\mathrm{inv}}^{2} v_{\max }}{2 h}\left\|\left[\Theta^{h}\right]\right\|_{\Gamma}^{2} \\
\leq \frac{5 v_{\max }}{4}\|\nabla \Phi\|_{0}^{2}+\frac{\delta}{2 h}\|[\Phi]\|_{\Gamma}^{2} \leq \frac{5 C_{0} v_{\max }}{4}\|\Phi\|_{1}^{2}+\frac{\tilde{C}_{\mathrm{tr}} \delta}{h}\left(\|\Phi\|_{0}\|\Phi\|_{1}\right),
\end{aligned}
$$

here $C_{0}$ is defined as a constant associated with the equivalence of two norms on $\Omega$, namely, $\|\nabla(\cdot)\|_{0} \leq C_{0}\|\cdot\|_{1}$.

From (19), we can see that if selecting $\delta \geq 4 \tilde{C}_{\text {inv }}^{2} v_{\max }$, by interpolation inequality A2

$$
\begin{aligned}
\frac{v_{\min }}{2}\left\|\nabla \Theta^{h}\right\|_{0}^{2} & \leq \frac{1}{2}\left\|\sqrt{v} \nabla \Theta^{h}\right\|_{0}^{2} \\
& \leq \frac{5 C_{0} v_{\max }}{4}\|\Phi\|_{1}^{2}+\frac{\tilde{C}_{\mathrm{tr}} \delta}{h}\left(\|\Phi\|_{0}\|\Phi\|_{1}\right) \\
& \leq\left(\frac{5 C_{0} v_{\max }}{4}+\tilde{C}_{\mathrm{tr}} \delta\right) C_{I}^{2}\|\mathbf{u}\|_{k+1, \Omega}^{2} h^{2 k}
\end{aligned}
$$

which yields

$$
\left\|\nabla \Theta^{h}\right\|_{0} \leq\left(\frac{5 C_{0}}{2} \frac{v_{\max }}{v_{\min }}+\frac{2 \tilde{C}_{\mathrm{tr}}}{v_{\min }} \delta\right)^{1 / 2} C_{I}\|\mathbf{u}\|_{k+1, \Omega} h^{k}
$$

Due to the triangle inequality and the interpolation inequality A2, we finally arrive at

$$
\left\|\nabla\left(\mathbf{u}-\mathbf{u}_{h}\right)\right\|_{0} \leq\left\|\nabla \Theta^{h}\right\|_{0}+\|\nabla \Phi\|_{0} \leq C h^{k},
$$

with

$$
C=\left[1+\left(\frac{5 C_{0}}{2} \frac{v_{\max }}{v_{\min }}+\frac{2 \tilde{C}_{\mathrm{tr}}}{v_{\min }} \delta\right)^{1 / 2}\right] C_{I}\|\mathbf{u}\|_{k+1, \Omega}
$$

Noting that in the estimate (22), $\delta$ appears in $C$, the right side of (22), this parameter $\delta$ cannot be too large to ensure a good approximation result. Compared with the term before $\delta$ in $C$, we can select

$$
4 \tilde{C}_{\mathrm{inv}}^{2} v_{\max } \leq \delta \leq \max \left\{\frac{5 C_{0} v_{\max }}{4 \tilde{C}_{\mathrm{tr}}}, 4 \tilde{C}_{\mathrm{inv}}^{2} v_{\max }\right\}
$$

The proof is completed. 
Remark 2 For the stabilization parameter $\delta$ selected above, we always have $C \leq[1+$ $\left.\left(5 C_{0} \frac{v_{\max }}{v_{\min }}\right)^{1 / 2}\right] C_{I}\|\mathbf{u}\|_{k+1, \Omega}$, which is independent of the mesh size $h$.

In the end, we present the error estimate in $L^{2}\left(\Omega_{i}\right)$ for the coupled scheme.

Theorem 2 Let $\mathbf{u}$ be the solution of the variational formulation (8) and $\mathbf{u}_{h}$ be the finite element solution deduced by Algorithm 1. Assuming that $\mathbf{u} \in X \cap H^{k+1}(\Omega)$, we have the following estimate:

$$
\left\|\mathbf{u}-\mathbf{u}_{h}\right\|_{0} \leq C h^{k+1} .
$$

Proof Firstly we construct a dual problem of (3) as follows:

$$
\begin{aligned}
& -\nabla \cdot\left(v_{i} \nabla z_{i}\right)=u_{i}-u_{i, h}, \quad \text { in } \Omega_{i}, i=1,2, \\
& z_{i}=0, \quad \text { on } \partial \Omega_{i} \backslash \Gamma, \\
& z_{1}-z_{2}=0, \quad \text { on } \Gamma, \\
& n_{1} \cdot v_{1} \nabla z_{1}+n_{2} \cdot v_{2} \nabla z_{2}=r, \quad \text { on } \Gamma .
\end{aligned}
$$

Choosing $r=0$, we have

$$
\begin{aligned}
\left\|\mathbf{u}-\mathbf{u}_{h}\right\|_{0}^{2}= & a\left(\mathbf{z}, \mathbf{u}-\mathbf{u}_{h}\right)+\frac{\delta}{h} \int_{\Gamma}[\mathbf{z}]\left[\mathbf{u}-\mathbf{u}_{h}\right] d s \\
& -\frac{1}{2} \int_{\Gamma}[v \nabla \mathbf{z} \cdot \mathbf{n}]\left[\mathbf{u}-\mathbf{u}_{h}\right] d s .
\end{aligned}
$$

Thanks to (13), we know that

$$
\begin{aligned}
\left\|\mathbf{u}-\mathbf{u}_{h}\right\|_{0}^{2}= & a\left(\mathbf{z}-I_{h} \mathbf{z}, \mathbf{u}-\mathbf{u}_{h}\right)+\frac{\delta}{h} \int_{\Gamma}\left[\mathbf{z}-I_{h} \mathbf{z}\right]\left[\mathbf{u}-\mathbf{u}_{h}\right] d s \\
& -\frac{1}{2}\left(\left[v \nabla\left(\mathbf{u}-\mathbf{u}_{h}\right) \cdot \mathbf{n}\right],\left[I_{h} \mathbf{z}\right]\right)_{\Gamma}-\frac{1}{2} \int_{\Gamma}[v \nabla \mathbf{z} \cdot \mathbf{n}]\left[\mathbf{u}-\mathbf{u}_{h}\right] d s .
\end{aligned}
$$

Noting that $\left[I_{h} \mathbf{z}\right]=0,[v \nabla \mathbf{z} \cdot \mathbf{n}]=0$ on $\Gamma$, by the Hölder inequality, the trace inequality $\mathrm{A} 1$ and the interpolation inequalities $\mathrm{A} 2$, we can obtain that

$$
\begin{aligned}
\left\|\mathbf{u}-\mathbf{u}_{h}\right\|_{0}^{2} & =a\left(\mathbf{z}-I_{h} \mathbf{z}, \mathbf{u}-\mathbf{u}_{h}\right)+\frac{\delta}{h} \int_{\Gamma}\left[\mathbf{z}-I_{h} \mathbf{z}\right]\left[\mathbf{u}-\mathbf{u}_{h}\right] d s \\
& \leq C\left\|\nabla\left(\mathbf{z}-I_{h} \mathbf{z}\right)\right\|_{0}\left\|\nabla\left(\mathbf{u}-\mathbf{u}_{h}\right)\right\|_{0}+\frac{C \delta}{h}\left\|\mathbf{z}-I_{h} \mathbf{z}\right\|_{L^{2}(\Gamma)}\left\|\mathbf{u}-\mathbf{u}_{h}\right\|_{L^{2}(\Gamma)} \\
& \leq C h\|\mathbf{z}\|_{2}\left\|\nabla\left(\mathbf{u}-\mathbf{u}_{h}\right)\right\|_{0}+C \delta h^{1 / 2}\|\mathbf{z}\|_{2}\left\|\mathbf{u}-\mathbf{u}_{h}\right\|_{0}^{1 / 2}\left\|\nabla\left(\mathbf{u}-\mathbf{u}_{h}\right)\right\|_{0}^{1 / 2} .
\end{aligned}
$$

Since the domain and the interface are both convex, we can derive from (26) that, while $r=0$,

$$
\|\mathbf{z}\|_{2} \leq C\left\|\mathbf{u}-\mathbf{u}_{h}\right\|_{0}
$$


Then we arrive at

$$
\begin{aligned}
\| \mathbf{u} & -\mathbf{u}_{h} \|_{0}^{2} \\
& \leq C h\left\|\mathbf{u}-\mathbf{u}_{h}\right\|_{0}\left\|\nabla\left(\mathbf{u}-\mathbf{u}_{h}\right)\right\|_{0}+C \delta h^{1 / 2}\left\|\mathbf{u}-\mathbf{u}_{h}\right\|_{0}^{3 / 2}\left\|\nabla\left(\mathbf{u}-\mathbf{u}_{h}\right)\right\|_{0}^{1 / 2} \\
& \leq C h^{2}\left\|\nabla\left(\mathbf{u}-\mathbf{u}_{h}\right)\right\|_{0}^{2}+\frac{1}{2}\left\|\mathbf{u}-\mathbf{u}_{h}\right\|_{0}^{2},
\end{aligned}
$$

which immediately yields

$$
\left\|\mathbf{u}-\mathbf{u}_{h}\right\|_{0} \leq C h^{k+1}
$$

The proof is completed.

\section{Numerical tests}

In this section, several numerical examples are carried out to illustrate our theoretical results. We select the stabilization parameter $\delta=1.0$ in the first three testing examples, and $\delta=0.1$ in the last case. The code is implemented and executed using the software package FreeFEM++ [28]. The linear solver UMFPACK is used for solving linear equations of the resulting system discretized by the finite element method for (10).

Example 1 Firstly we consider a stationary heat conduction problem in a single solving domain $\Omega=[0,1] \times[0,2]$ with analytic solution as follows:

$$
u=(2-\pi \sin (\pi x))(1-y-\cos (\pi y))
$$

By introducing a straight internal boundary $\Gamma=(0,1) \times\{1\}$, we divide the domain $\Omega$ into two subdomains $\Omega_{1}=[0,1] \times[0,1]$ and $\Omega_{2}=[0,1] \times[1,2]$. In this example $g=0$ in (3), $v_{1}=v_{2}$, and the right hand sides $f_{i}$ and other boundary conditions are derived from the exact solution. To this end, we can say that the present algorithm can be regarded as a special domain decomposition method for this problem.

A sequence of uniform triangular meshes are generated by aligning with the interface $\Gamma$. Based on these meshes, the $H^{1}$ and $L^{2}$ errors for numerical solutions by the present method are reported in Table 1 with $P_{1}(k=1$, linear $)$ and $P_{2}(k=2$, quadratic) elements. From this table and the plotted convergence results in Fig. 2, the numerical orders of $k$ and $k+1$ respectively in the sense of $H^{1}$ and $L^{2}$ norms are observed clearly.

Noting that in general, $u_{1}-u_{2}=\psi$ is usually not zero. However, in the non-zero case, we only need to add another additional term in the right hand side related to $\psi$, which will not obviously affect our analysis since $\psi$ is some prescribed function. To this end, we follow [29] in the next three examples to carry out our testings to verify theoretical results. For simplicity, we only consider the $P_{1}$ element hereafter.

Example 2 Now we consider a circular interface problem. The domain $\Omega_{1}$ consists of a circle with its center at the origin and a radius of 0.5 . Let $\Omega=[-1,1] \times[-1,1]$ and $\Omega_{2}=\Omega \backslash$ $\Omega_{1}$. The coefficients $v_{i}$ for different domains $\Omega_{i}$ are chosen as $v_{1}=10, v_{2}=1$, respectively. The analytical solution is chosen as

$$
u_{1}=10-x^{2}-y^{2}, \quad u_{2}=\sin (\pi x) \sin (\pi y) .
$$


Table 1 Convergence order with respect to $h$ of the stabilized method for Example 1 with $P_{1}$ and $P_{2}$ elements

\begin{tabular}{lllll}
\hline & $\left\|\mathbf{u}-\mathbf{u}_{h}\right\|_{0}$ & Order $(h)$ & $\left\|\mathbf{u}-\mathbf{u}_{h}\right\|_{1}$ & Order $(h)$ \\
\hline$h\left(P_{1}\right)$ & & & & \\
$\frac{1}{16}$ & $1.04210 \mathrm{e}-1$ & - & 2.70631 & - \\
$\frac{1}{32}$ & $2.68904 \mathrm{e}-2$ & 1.954 & 1.36429 & 0.988 \\
$\frac{1}{64}$ & $6.79800 \mathrm{e}-3$ & 1.984 & 0.683551 & 0.997 \\
$\frac{1}{128}$ & $1.70751 \mathrm{e}-3$ & 1.993 & 0.341947 & 0.999 \\
$h\left(P_{2}\right)$ & & & & \\
$\frac{1}{8}$ & $3.10623 \mathrm{e}-2$ & - & 0.823449 & - \\
$\frac{1}{16}$ & $4.63642 \mathrm{e}-3$ & 2.744 & 0.209248 & 1.976 \\
$\frac{1}{32}$ & $6.15439 \mathrm{e}-4$ & 2.913 & 0.0522218 & 2.002 \\
$\frac{1}{64}$ & $7.83590 \mathrm{e}-5$ & 2.973 & 0.0130404 & 2.002 \\
\hline
\end{tabular}
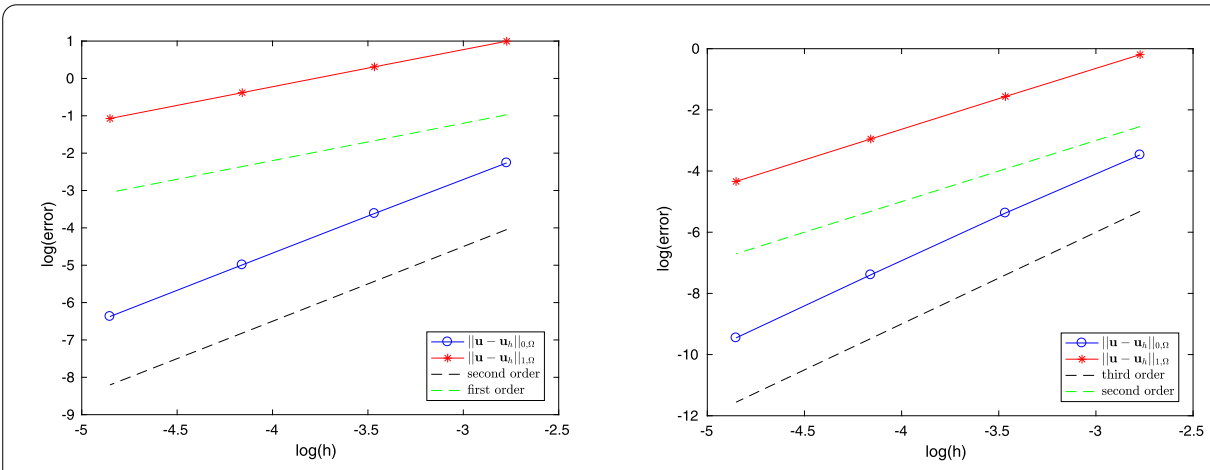

Figure 2 Plots of orders of convergence for Example 1. Left: $P_{1}$ elements; Right: $P_{2}$ elements

Table 2 Convergence order with respect to $h$ of the stabilized method for Example 2

\begin{tabular}{lllll}
\hline$h$ & $\left\|\mathbf{u}-\mathbf{u}_{h}\right\|_{0}$ & $\operatorname{Order}(h)$ & $\left\|\mathbf{u}-\mathbf{u}_{h}\right\|_{1}$ & Order $(h)$ \\
\hline$\frac{1}{16}$ & $6.02324 \mathrm{e}-2$ & - & $6.43806 \mathrm{e}-1$ & - \\
$\frac{1}{32}$ & $1.64189 \mathrm{e}-2$ & 1.885 & $3.22031 \mathrm{e}-1$ & 1.005 \\
$\frac{1}{64}$ & $4.16997 \mathrm{e}-3$ & 1.996 & $1.62769 \mathrm{e}-1$ & 0.994 \\
$\frac{1}{128}$ & $1.14220 \mathrm{e}-3$ & 1.861 & $8.15625 \mathrm{e}-2$ & 0.993 \\
$\frac{1}{256}$ & $3.02782 \mathrm{e}-4$ & 1.904 & $4.07209 \mathrm{e}-2$ & 0.996 \\
\hline
\end{tabular}

The numerical results of the stabilized method are listed in Table 2, the computed orders of convergence are plotted in Fig. 3. As $h$ decreases, it is easy to see that the numerical convergence orders are very close to 2 in $L^{2}$ norm and 1 in $H^{1}$ norm, respectively. These numerical results are consistent with our theoretical ones as proved in Theorem 1 and Theorem 2.

Example 3 In this example, we select $\Omega=[0,1] \times[0,1]$ and $\Omega_{1}=[0.2,0.8] \times[0.2,0.8]$ with $\Omega_{2}=\Omega \backslash \Omega_{1}$. The coefficient functions $v_{i}$ and the analytic solutions $u_{i}$ in different subdomains are chosen as the following bounded functions:

$$
\begin{aligned}
& v_{1}=1, \quad v_{2}=2+\sin (x+y) ; \\
& u_{1}=5+5\left(x^{2}+y^{2}\right), \quad u_{2}=x^{2}+y^{2}+\sin (x+y) .
\end{aligned}
$$


Figure 3 Plots of orders of convergence for Example 2 by $P_{1}$ elements

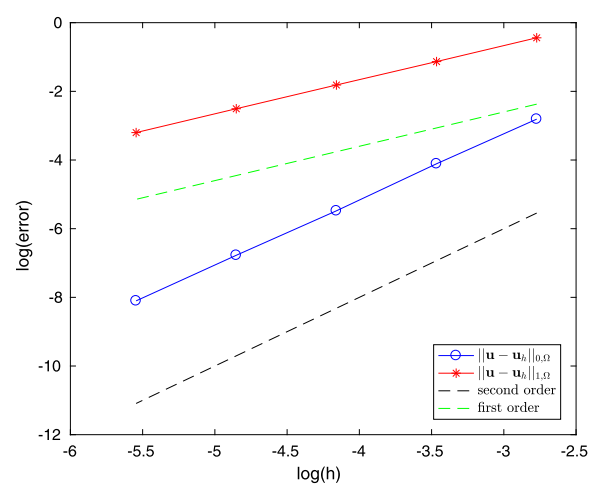

Table 3 Convergence order with respect to $h$ of the stabilized method for Example 3 with coefficient functions

\begin{tabular}{lllll}
\hline$h$ & $\left\|\mathbf{u}-\mathbf{u}_{h}\right\|_{0}$ & $\operatorname{Order}(h)$ & $\left\|\mathbf{u}-\mathbf{u}_{h}\right\|_{1}$ & Order $(h)$ \\
\hline$\frac{1}{16}$ & $4.14523 \mathrm{e}-3$ & - & $1.78752 \mathrm{e}-1$ & - \\
$\frac{1}{32}$ & $8.37080 \mathrm{e}-4$ & 2.313 & $9.03064 \mathrm{e}-2$ & 0.987 \\
$\frac{1}{64}$ & $1.91750 \mathrm{e}-4$ & 2.114 & $4.41597 \mathrm{e}-2$ & 1.026 \\
$\frac{1}{128}$ & $4.17206 \mathrm{e}-5$ & 2.144 & $2.22359 \mathrm{e}-2$ & 0.964 \\
$\frac{1}{256}$ & $1.00927 \mathrm{e}-5$ & 2.078 & $1.11664 \mathrm{e}-2$ & 1.008 \\
\hline
\end{tabular}

Figure 4 Plots of orders of convergence for Example 3 by $P_{1}$ elements

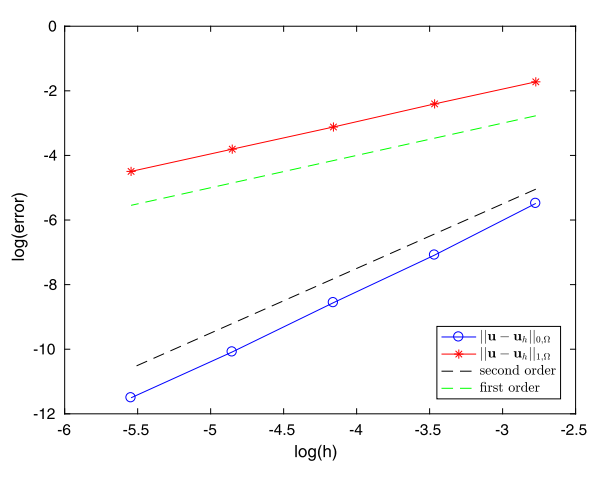

The approximate results are shown in Table 3, with successive mesh refinements, and also plotted in Fig. 4. Obviously, the theoretical orders, i.e., $O\left(h^{2}\right)$ in $L^{2}$ norm and $O(h)$ in $H^{1}$ norm, respectively, are verified based on such $P_{1}$ elements, as provided in Theorem 1 and Theorem 2.

Example 4 As the last testing case, we consider a problem on domain $\Omega=[0,1]^{2}$ with an interface of flower petal, whose parametric form is given as (cf. Example 9 of Ref. [9]):

$$
\begin{aligned}
& x(\theta)=(a+b \cos (m \theta)) \sin (n \theta) \cos (\theta), \\
& y(\theta)=(a+b \cos (m \theta)) \sin (n \theta) \sin (\theta), \quad \theta \in[0,2 \pi] .
\end{aligned}
$$


Table 4 Convergence order with respect to $h$ of the stabilized method for Example 4 with coefficient functions

\begin{tabular}{lllllllr}
\hline$h$ & $\left\|\mathbf{u}-\mathbf{u}_{h}\right\|_{0}$ & $\operatorname{Order}(h)$ & $\left\|\mathbf{u}-\mathbf{u}_{h}\right\|_{1}$ & $\operatorname{Order}(h)$ & $\left\|\mathbf{u}-\mathbf{u}_{h}\right\|_{0, \infty}$ & $\operatorname{Order}(h)$ & CPU $(s)$ \\
\hline$\frac{1}{16}$ & $4.39386 \mathrm{e}-3$ & - & $3.84330 \mathrm{e}-2$ & - & $8.04136 \mathrm{e}-3$ & - & 0.725 \\
$\frac{1}{32}$ & $1.25230 \mathrm{e}-3$ & 1.870 & $1.85753 \mathrm{e}-2$ & 1.083 & $2.49653 \mathrm{e}-3$ & 1.742 & 1.609 \\
$\frac{1}{64}$ & $2.30183 \mathrm{e}-4$ & 2.441 & $8.35445 \mathrm{e}-3$ & 1.151 & $5.91155 \mathrm{e}-4$ & 2.076 & 7.721 \\
$\frac{1}{128}$ & $6.05530 \mathrm{e}-5$ & 1.923 & $4.01170 \mathrm{e}-3$ & 1.057 & $1.45916 \mathrm{e}-4$ & 2.015 & 29.335 \\
$\frac{1}{256}$ & $1.48867 \mathrm{e}-5$ & 2.008 & $1.95483 \mathrm{e}-3$ & 1.029 & $3.48517 \mathrm{e}-5$ & 2.050 & 99.845 \\
\hline
\end{tabular}

Figure 5 Plots of orders of convergence for Example 4 by $P_{1}$ elements

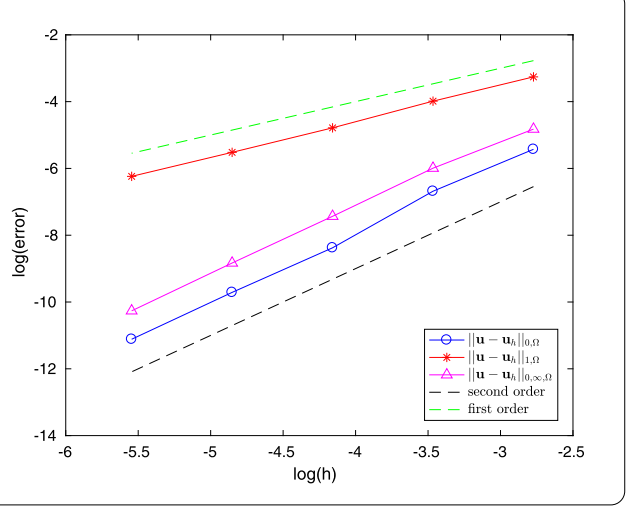

Here we take $a=0.50012563, b=0.250012563, m=0$, and $n=10$. The coefficient function and the analytical solution are chosen as

$$
\begin{array}{ll}
v_{1}=(x y+2) / 5, & v_{2}=\left(x^{2}-y^{2}+3\right) / 7 \\
u_{1}=x+y+1, & u_{2}=\sin (x+y)+\cos (x+y)+1 .
\end{array}
$$

For this testing case, the error in $L^{2}, H^{1}$ and $L^{\infty}$ norms and corresponding CPU time are listed in Table 4. Meanwhile, the numerical orders of convergence with respect to decreasing mesh size $h$ are plotted in Fig. 5. We can observe how the optimal convergence results emerge.

\section{Conclusions}

In this paper we have proposed a stabilized coupled algorithm for the elliptic interface problem. The main contribution of the present work is the analysis of the optimal error estimates for the present algorithm. Due to its simplicity of scheme construction, it can be generalized to even higher-order accuracy schemes for more complicated interfaces related to time and space. Several numerical experiments have also been conducted to demonstrate the computational stability and effectiveness of the present algorithm. In the next step we will be concerned with some moving interface problems for more complicated fluid models. 
Availability of data and materials

Please contact the author for data requests.

\section{Competing interests}

The authors declare that they have no competing interests.

\section{Authors' contributions}

The authors declare that the study was realized in collaboration with equal responsibility. All authors read and approved the final manuscript.

\section{Author details}

${ }^{1}$ College of Science, Donghua University, Shanghai, China. ${ }^{2}$ Institute for Nonlinear Sciences, Donghua University, Shanghai, China. ${ }^{3}$ College of Science, Harbin Institute of Technology, Shenzhen, China. ${ }^{4}$ College of Mathematics and Systems Science, Xinjiang University, Urumqi, China. ${ }^{5}$ Institute of mathematics and Physics, Xinjiang University, Urumqi, China.

\section{Publisher's Note}

Springer Nature remains neutral with regard to jurisdictional claims in published maps and institutional affiliations.

\section{Received: 3 May 2019 Accepted: 5 September 2019 Published online: 18 September 2019}

\section{References}

1. Peskin, C.S.: Numerical analysis of blood flow in heart. J. Comput. Phys. 25, 220-252 (1977)

2. Leveque, R.J., Li, Z.: The immersed interface method for elliptic equations with discontinuous coefficients and singular sources. SIAM J. Numer. Anal. 31, 1019-1044 (1994)

3. Berthelsen, P.A.: A decomposed immersed interface method for variable coefficient elliptic equations with non-smooth and discontinuous solutions. J. Comput. Phys. 197, 364-386 (2004)

4. Yu, S., Zhou, Y., Wei, G.: Matched interface and boundary (MIB) method for elliptic problems with sharp-edged interfaces. J. Comput. Phys. 224, 729-756 (2007)

5. Zhao, J., Hou, Y., Li, Y.: Immersed interface method for elliptic equations based on a piecewise second order polynomial. Comput. Math. Appl. 63, 957-965 (2012)

6. Xia, J., Li, Z., Ye, X.: Effective matrix-free preconditioning for the augmented immersed interface method. J. Comput. Phys. 303, 295-312 (2015)

7. Li, Z., Lin, T., Lin, Y., Rogers, R.: An immersed finite element space and its approximation capability. Numer. Methods Partial Differ. Equ. 20, 338-367 (2004)

8. Gong, Y., Li, B., Li, Z.: Immersed-interface finite-element methods for elliptic interface problems with non-homogeneous jump conditions. SIAM J. Numer. Anal. 46, 472-495 (2008)

9. Mu, L., Wang, J., Ye, X., Zhao, S.: A new weak Galerkin finite element method for elliptic interface problems. J. Comput. Phys. 325, 157-173 (2016)

10. Fries, T.P., Belytschko, T.: The extended-generalized finite element method-an overview of the method and its applications. Int. J. Numer. Methods Eng. 84, 253-304 (2010)

11. Zhao, J., Hou, Y., Song, L.: Modified intrinsic extended finite element method for elliptic equation with interfaces. J. Eng. Math. 97, 1-13 (2016)

12. Babuška, I., Banerjee, U.: Stable generalized finite element method (SGFEM). Comput. Methods Appl. Mech. Eng. 201, 91-111(2012)

13. Hou, S., Song, P., Wang, L., Zhao, H.: A weak formulation for solving elliptic interface problems without body fitted grid. J. Comput. Phys. 249, 80-95 (2013)

14. Hou, S., Wang, L., Shi, L.: An improved non-traditional finite element formulation for solving the elliptic interface problems. Comput. Math. Appl. 73, 374-384 (2017)

15. Bernardi, C., Verfürth, R.: Adaptive finite element methods for elliptic equations with non-smooth coefficients. Numer Math. 85, 579-608 (2000)

16. Morin, P., Nochetto, R.H., Siebert, K.G.: Convergence of adaptive finite element methods. SIAM Rev. 44, 631-658 (2002)

17. Cai, Z., Zhang, S.: Recovery-based error estimator for interface problems: conforming linear elements. SIAM J. Numer. Anal. 47, 2132-2156 (2009)

18. Chen, Z., Zou, J.: Finite element methods and their convergence for elliptic and parabolic interface problems. Numer. Math. 79, 457-492 (1998)

19. Yu, J., Shi, F., Zheng, H.: Local and parallel finite element method based on the partition of unity for the Stokes problem. SIAM J. Sci. Comput. 36, 547-567 (2014)

20. Zheng, H., Shi, F., Hou, Y., Zhao, J., Cao, Y., Zhao, R.: A new local and parallel finite element algorithm based on the partition of unity. J. Math. Anal. Appl. 435, 1-19 (2016)

21. Shan, L., Zheng, H.: Partitioned time stepping method for fully evolutionary Stokes-Darcy flow with Beavers-Joseph interface conditions. SIAM J. Numer. Anal. 51, 813-839 (2013)

22. Hou, Y.: Optimal error estimates of a decoupled scheme based on two-grid finite element for mixed Stokes-Darcy model. Appl. Math. Lett. 57, 90-96 (2016)

23. Zhang, Y., Hou, Y., Shan, L.: Stability and convergence analysis of a decoupled algorithm for a fluid-fluid interaction problem. SIAM J. Numer. Anal. 54, 2833-2867 (2016)

24. Layton, W., Schieweck, F. Yotov, I: Coupling fluid flow with porous media flow. SIAM J. Numer. Anal 40, 2195-2218 (2002)

25. Adams, R.A.: Sobolev Spaces. Academic Press, New York (1975)

26. Brenner, S., Scott, R.: The Mathematical Theory of Finite Element Methods. Springer, Berlin (2007) 
27. Galdi, G.P.: An Introduction to the Mathematical Theory of the Navier-Stokes Equations: Steady-State Problems. Springer, Berlin (2011)

28. Hecht, F., Le Hyaric, A., Ohtsuka, K., Pironneau, O.: Freefem++, Finite elements software. http://www.freefem.org/ff++/

29. Mu, M., Xu, J.: A two-grid method of a mixed Stokes-Darcy model for coupling fluid flow with porous media flow. SIAM J. Numer. Anal. 45, 1801-1813 (2007)

Submit your manuscript to a SpringerOpen ${ }^{\odot}$ journal and benefit from:

- Convenient online submission

- Rigorous peer review

- Open access: articles freely available online

- High visibility within the field

- Retaining the copyright to your article

Submit your next manuscript at $\gg$ springeropen.com 